\title{
Impact of Nonlinear Transfer Function and Imperfect Splitting Ratio of MZM on Optical Up-Conversion Employing Double Sideband With Carrier Suppression Modulation
}

\author{
Chun-Ting Lin, Jason (Jyehong) Chen, Sheng-Peng Dai, Peng-Chun Peng, and Sien Chi, Fellow, OSA
}

\begin{abstract}
Generation of optical millimeter-wave (mm-wave) signal using a Mach-Zehnder modulator (MZM) based on double-sideband (DSB), single-sideband (SSB), and double-sideband with carrier suppression (DSBCS) modulation schemes have been demonstrated for various applications, such as broadband wireless signals or optical up-conversion for wavelength-division-multiplexing (WDM) radio-over-fiber (RoF) network, wideband surveillance, spread spectrum, and software-defined radio. Among these schemes, DSBCS modulation offers the best receiver sensitivity, lowest spectral occupancy, the least stringent requirement of electrical bandwidth, and the smallest receiving power penalty after long transmission distance. Nonetheless, the inherent nonlinear $\mathrm{E} / \mathrm{O}$ (electrical/optical) conversion response of a MZM is such that the signal quality of the optical mm-wave suffers. Fabrication tolerances make a balanced 50/50 splitting ratio of the MZM's $y$-splitter particularly difficult to achieve. As a result, imbalanced MZMs have a finite extinction ratio (ER) and degrade the optical carrier suppression ratio (OCSR) using DSBCS modulation. In this paper, the effect of the MZM nonlinearity and imbalanced $y$-splitter on optical mm-wave generation by DSBCS modulation is theoretically and experimentally investigated. A novel approach with better performance and greater cost-effectiveness than dual-electrode MZM (DD-MZM) is presented to realize a DSBCS modulation scheme based on a single-electrode MZM (SD-MZM).
\end{abstract}

Index Terms-Microwave photonics, millimeter-wave (mmwave) generation, Mach-Zehnder modulator (MZM), MZM imbalance, MZM nonlinearity, radio-over-fiber (RoF).

\section{INTRODUCTION}

$\mathbf{T}$ HE pervasiveness of handheld devices has lead to rapid growth in the demand on broadband wireless communication. However, insufficient bandwidth and serious propagation loss make traditional coaxial cable unsuitable for the

Manuscript received January 9, 2008; revised May 1, 2008. Current version published October 10, 2008. This work was supported by the National Science Council of R.O.C. under Contract NSC 96-2221-E-155-038-MY2, NSC 96-2752-E-009-004-PAE, and NSC 96-2628-E-009-016.

C.-T. Lin, J. (J.) Chen, and S.-P. Dai, are with the Department of Photonics and Institute of Electro-Optical Engineering, National Chiao-Tung University, Hsinchu, Taiwan 300, R.O.C. (e-mail: jinting@ms94.url.com.tw).

P.-C. Peng is with the Department of Applied Materials and Optoelectronic Engineering, National Chi Nan University, Taiwan 545, R.O.C.

S. Chi is with the Department of Photonics and Institute of Electro-Optical Engineering, National Chiao-Tung University, Hsinchu, Taiwan 300, R.O.C., and also with the Department of Electrical Engineering, Yuan-Ze University, Chung Li, Taiwan 320, R.O.C.

Digital Object Identifier 10.1109/JLT.2008.927160 transmission of wireless signals in the microwave/millimeterwave (mm-wave) range. Therefore, the radio-over-fiber $(\mathrm{RoF})$ system, which distributes radio-frequency (RF) signals from a central station (CS) to base stations (BS) over an optical fiber, is a promising approach because of its almost unlimited bandwidth and very low propagation loss [1]-[3].

RoF technology allows the concentration of RF signal processing and shared mm-wave components at a CS and makes BS simpler and more cost-effective. The generation and transmission of optical microwave or mm-wave signals are crucial to RoF systems. Optical RF signal generation or optical up-conversion using an external Mach-Zehnder modulator (MZM) based on double-sideband (DSB), single-sideband (SSB), and double-sideband with carrier suppression (DSBCS) modulation schemes have been demonstrated [3]-[7]. The DSB modulation signal undergoes performance fading problems because of fiber dispersion, which causes periodic degradation of the receiver sensitivity varying with the transmission length of standard single mode fiber (SSMF). The SSB modulation, which is generated by applying a $\pi / 2$ phase difference between the two RF electrodes of the dual-electrode MZM (DD-MZM) biased at the quadrature point, is developed to overcome the performance fading. However, since the optical RF signals are weakly modulated because of the narrow linear region of MZM, those that undergo DSB and SSB modulations have inferior sensitivities because the optical modulation depth (OMD) is limited [4]. Recently, the DSBCS modulation has been demonstrated in the $\mathrm{mm}$-wave range to have the best receiver sensitivity and overcome periodic performance fading due to fiber dispersion. Besides, since optical carrier is suppressed, a frequency doubling technique can be achieved to reduce the bandwidth requirement of optical RF transmitter [5].

However, independent of the modulation scheme employed, MZM modulation is associated with an inherently nonlinear E/O conversion response. Hence, the MZM nonlinear distortion negatively affects the performance of the mm-wave signal. Besides, fabrication tolerances make a balanced 50/50 splitting ratio of the MZM's Y-splitter particularly difficult to achieve. Therefore, imbalanced MZMs have a finite extinction ratio (ER) and the mm-wave signals based on DSBCS modulation have finite optical carrier suppression ratio (OCSR), degrading the quality of mm-wave signals. Although numerous studies of the nonlinear distortion of $\mathrm{mm}$-wave signals generated using the DSB and SSB modulation approaches have been recently 


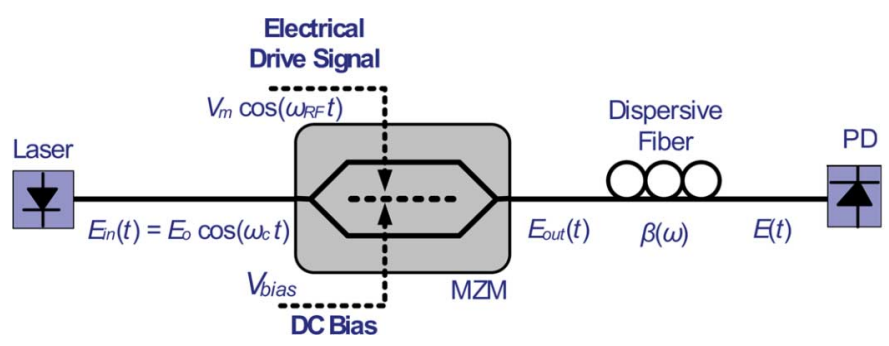

Fig. 1. The principle diagram of the optical mm-wave generation using balanced MZM based on DSBCS modulation.

conducted [8]-[11], to the author's best knowledge, no information is available on the combined effects of MZM nonlinear distortion and imbalance on mm-wave generation or optical up-conversion by the DSBCS modulation scheme. This investigation theoretically and experimentally analyzes the impacts of both MZM nonlinearity and imbalance on the mm-wave signal performance. Optimal conditions for optical mm-wave generation using imbalanced MZM based on DSBCS modulation are discussed. In conventional DSBCS modulation scheme, a full swing ( $2 \mathrm{~V} \pi$, typically $10-14 \mathrm{~V}$ ) electrical RF driving signal is necessary to ensure high OCSR. The limited driving capability of the electrical amplifier requires a push-pull DD-MZM to be used to weaken the demanding driving voltage requirement. This limitation increases the system complexity and cost because extra and more expensive components are needed. This work presents a novel method that uses a single-electrode MZM (SD-MZM) to generate the mm-wave signal with performance improvement based on DSBCS modulation scheme. Both theoretical and experimental results show that the proposed system is compact, cost-effective, and performs better.

\section{Theoretical Analysis of Millimeter-WaVe GENERATION BY DSBCS MODULATION}

\section{A. Generation of mm-Wave Signal Using Balanced MZM}

Fig. 1 displays the principle of the optical mm-wave generation by the DSBCS modulation scheme. The power splitting ratio of two arms of a balanced MZM is 0.5. The electrical field at the output of the MZM is given by

$$
E_{\text {out }}(t)=E_{o} \cos \left[\frac{\Phi[V(t)]}{2}\right] \cos \left(\omega_{c} t\right)
$$

where $E_{0}$ and $\omega_{c}$ denote the amplitude and angular frequency of the input optical carrier, respectively, $V(t)$ is the applied driving voltage, and $\Phi[V(t)]$ is the optical carrier phase difference that is induced by $V(t)$ between the two arms of the MZM. The loss of MZM is neglected. $V(t)$ consisting of an electrical sinusoidal signal and a dc biased voltage can be written as

$$
V(t)=V_{\text {bias }}+V_{m} \cos \left(\omega_{\mathrm{RF}} t\right)
$$

where $V_{\text {bias }}$ is the dc biased voltage, and $V_{m}$ and $\omega_{\mathrm{RF}}$ are the amplitude and the angular frequency of the electrical driving signal, respectively. The optical carrier phase difference induced by $V(t)$ is given by

$$
\Phi[V(t)]=\pi\left[\frac{V_{\mathrm{bias}}}{V_{\pi}}+\frac{V_{m}}{V_{\pi}} \cos \left(\omega_{\mathrm{RF}} t\right)\right]
$$

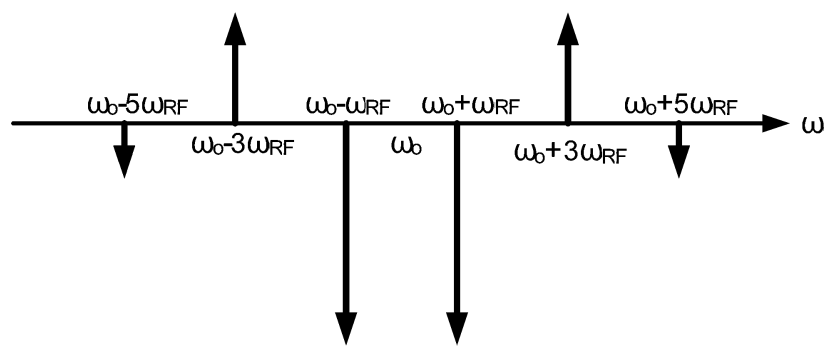

Fig. 2. Illustration of the optical spectrum at the output of the balanced MZM.

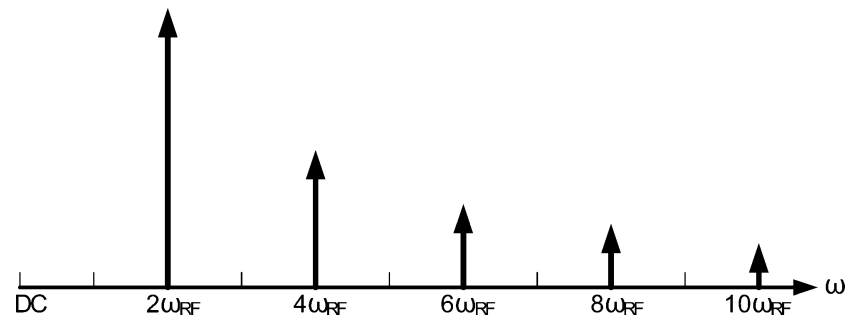

Fig. 3. Illustration of the electrical spectrum of the mm-wave signal using balanced MZM after square-law PD detection.

where $V_{\pi}$ is the half-wave voltage of the MZM. Therefore, the output electrical field can be rewritten as

$$
\begin{aligned}
E_{\text {out }}(t)= & E_{o} \cos \left(\frac{\pi}{2}\left[\frac{V_{\text {bias }}}{V_{\pi}}+\frac{V_{m}}{V_{\pi}} \cos \left(\omega_{\mathrm{RF}} t\right)\right]\right) \cos \left(\omega_{c} t\right) \\
= & E_{o}\left\{\cos \mathrm{b} \cdot \cos \left[\mathrm{m} \cos \left(\omega_{\mathrm{RF}} t\right)\right]\right. \\
& \left.-\sin \mathrm{b} \cdot \sin \left[\mathrm{m} \cos \left(\omega_{\mathrm{RF}} t\right)\right]\right\} \cdot \cos \left(\omega_{c} t\right)
\end{aligned}
$$

where $\mathrm{b} \stackrel{\text { def }}{=}\left(V_{\text {bias }} / 2 V_{\pi}\right) \pi$ is a constant phase shift that is induced by the dc biased voltage, and $\mathrm{m} \stackrel{\text { def }}{=}\left(V_{m} / 2 V_{\pi}\right) \pi$ is the phase modulation index. Expanding (4) using Bessel functions, as detailed in Appendix I, yields Fig. 2, which illustrates the optical spectrum of the mm-wave signals obtained by DSBCS modulation with the MZM biased at the null point. All of the even-order optical sidebands are eliminated, so only the odd-order optical sidebands remain in the spectrum. Square-law photo-diode (PD) detection yields the corresponding electrical spectrum of the generated $\mathrm{mm}$-wave signals which are shown in Fig. 3. No original driving signal $\omega_{R F}$ and the odd terms of its harmonics distortions $\left(3 \omega_{\mathrm{RF}}, 5 \omega_{\mathrm{RF}}, 7 \omega_{\mathrm{RF}} \ldots\right)$ exist. A strong anticipated double-frequency signal $\left(2 \omega_{\mathrm{RF}}\right)$ and the odd terms of the harmonic distortions $\left(4 \omega_{\mathrm{RF}}, 6 \omega_{\mathrm{RF}}, 8 \omega_{\mathrm{RF}} \cdots\right)$ are observed.

\section{B. Generation of mm-Wave Signal Using Imbalanced MZM}

Fig. 4 shows the MZM with an imbalanced power splitting ratio of the Y-splitter. The electrical field at the output of the MZM can be written as

$$
\begin{array}{r}
E_{\text {out }}(t)=\Re e\left\{E _ { o } \left[\sqrt{r_{1}} \cdot \sqrt{r_{2}} \cdot \exp \left(j \varphi_{1}\right)+\sqrt{1-r_{1}}\right.\right. \\
\left.\left.\cdot \sqrt{1-r_{2}} \cdot \exp \left(j \varphi_{2}\right)\right] \cdot \exp \left(j \omega_{c} t\right)\right\}
\end{array}
$$

where $r_{1}$ and $r_{2}$ are the power splitting ratios of the first and second Y-splitters in MZM, respectively; $\varphi_{1}$ and $\varphi_{2}$ are the optical carrier phase shifts induced by the applied driving voltages 


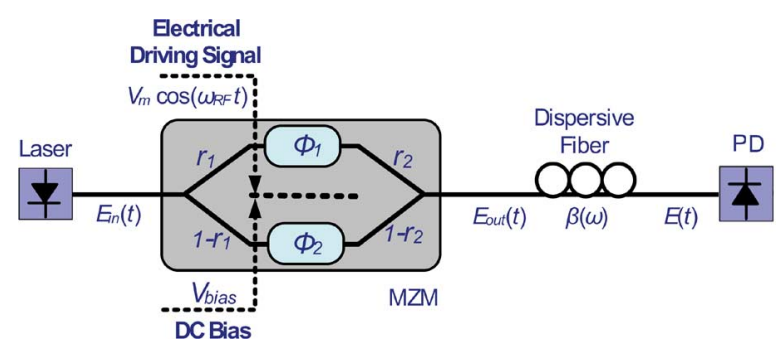

Fig. 4. The principle diagram of the optical mm-wave generation using imbalanced MZM based on DSBCS modulation.

in the upper and lower arms of the MZM, respectively. The ER of the imbalanced MZM can be expressed as

$$
\mathrm{ER}=\left[\frac{\sqrt{r_{1}} \cdot \sqrt{r_{2}}+\sqrt{1-r_{1}} \cdot \sqrt{1-r_{2}}}{\sqrt{r_{1}} \cdot \sqrt{r_{2}}-\sqrt{1-r_{1}} \cdot \sqrt{1-r_{2}}}\right]^{2}
$$

Notably, the MZM ER is infinite as $r_{1}=r_{2}=0.5$ or $r_{1}=$ $1-r_{2}$. If not, the MZM ER will have a finite value. Without loss of generality, the assumption that $\sqrt{r_{1}} \cdot \sqrt{r_{2}}<\sqrt{1-r_{1}}$. $\sqrt{1-r_{2}}$, enables (6) to be rewritten as

$$
\begin{aligned}
E_{\text {out }}(t)= & \operatorname{Re}\left\{\sqrt { r _ { 1 } } \cdot \sqrt { r _ { 2 } } \cdot \left[\exp \left(j \varphi_{1}\right)\right.\right. \\
& \left.+\exp \left(j \varphi_{2}\right)\right] \cdot \exp \left(j \omega_{c} t\right)+E_{o}\left[\sqrt{1-r_{1}} \cdot \sqrt{1-r_{2}}\right. \\
& \left.\left.-\sqrt{r_{1}} \cdot \sqrt{r_{2}}\right] \cdot \exp \left(j \varphi_{2}\right) \cdot \exp \left(j \omega_{c} t\right)\right\} .
\end{aligned}
$$

Accordingly, the modulation of the MZM with imbalanced power splitting ratios can be regarded as the sum of a balanced MZM modulation plus a modulation by an extra phase modulator (PM) modulation which are represented by the first and second terms of (7), respectively. Evidently, for a balanced MZM with $r_{1}=r_{2}=0.5$ or $r_{1}=1-r_{2}$, no phase modulation term exists. However, fabrication tolerances make both conditions difficult to achieve. Thus, the inherent PM modulation that is caused by the imbalanced splitting ratios of the MZM cannot be avoided. Expanding (7), as detailed in Appendix II, yields the optical spectrum of the mm-wave signal using DSBCS modulation that is displayed in Fig. 5. A comparison with Fig. 3 indicates that the main effects of the undesired PM modulation, caused by imbalanced MZMs, are the generation of the superfluous optical carrier $\omega_{c}$ and even-order sidebands $\left(\omega_{c} \pm 2 \omega_{\mathrm{RF}}, \omega_{c} \pm 4 \omega_{\mathrm{RF}} \cdots\right)$. Additionally, the evenand odd-order terms are mutually orthogonal. Fig. 6(a) and (b) present back-to-back (BTB) and following fiber transmission electrical spectra of the generated $\mathrm{mm}$-wave signals. Notably, only the even-order BTB mm-wave signals are observed as shown in Fig. 6(a). However, after transmission over a dispersive fiber, the even- and odd-order terms are no longer mutually orthogonal because of fiber dispersion. Therefore, following square-law PD detection, the cross-terms generate the undesired odd-order signals $\left(3 \omega_{\mathrm{RF}}, 5 \omega_{\mathrm{RF}}, 7 \omega_{R F} \cdots\right)$ and also produce unwanted signals fall inside the target $2 \omega_{\mathrm{RF}} \mathrm{mm}$-wave band.

\section{Optical Carrier and Distortion Suppression Analysis}

An RoF lightwave system primarily consists of transmitters, a fiber link, and receivers. One of the key issues that govern

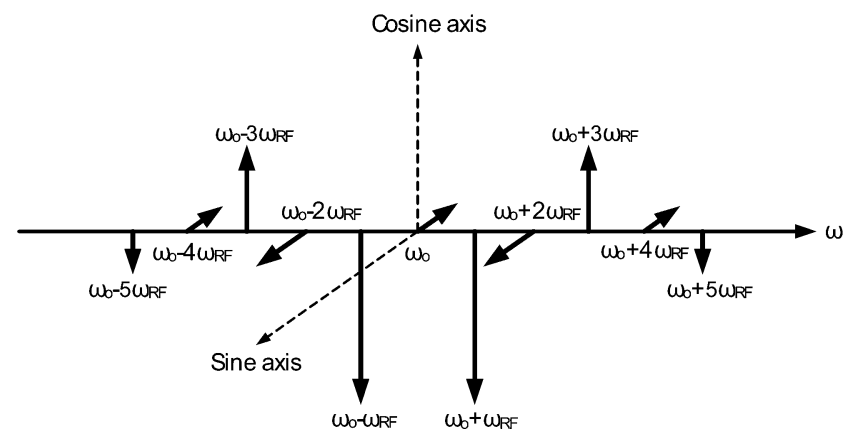

Fig. 5. Illustration of the optical spectrum at the output of the imbalanced MZM.

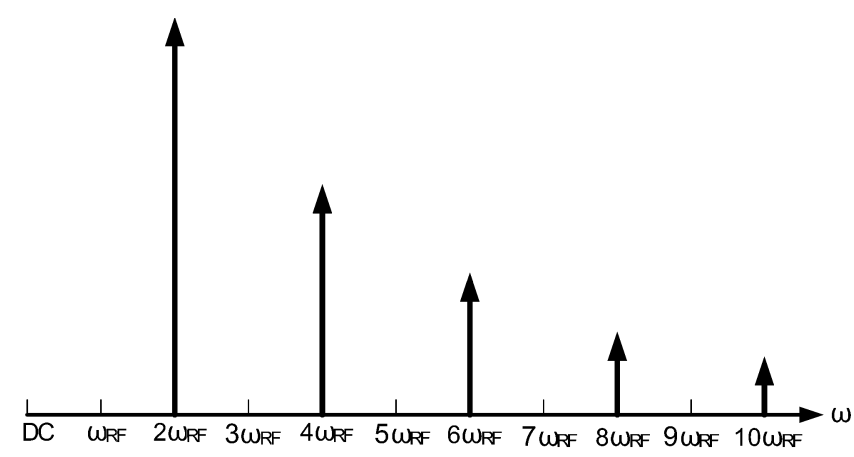

(a)

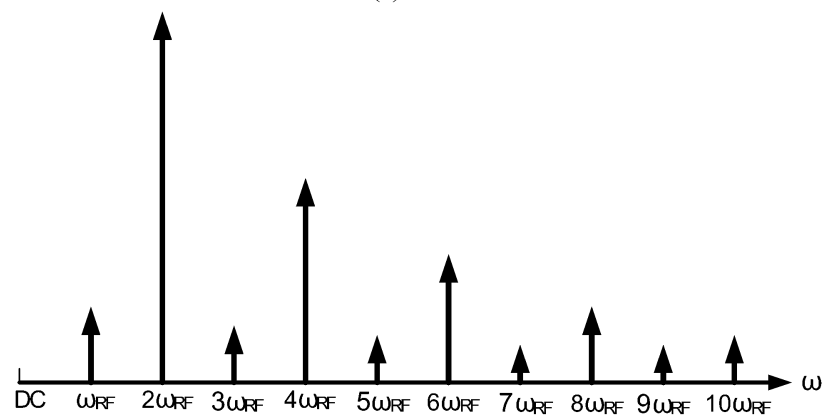

(b)

Fig. 6. Illustration of the electrical spectrum of generated mm-wave signals using imbalanced MZM after square-law PD detection. (a) BTB mm-wave signals. (b) Mm-wave signals after transmission over dispersion fiber.

the performance of the RoF system is the linearity of E/O conversion especially for external MZM modulation. The inherent nonlinearity of MZM makes the undesired optical sidebands of the mm-wave signals $\left(\omega_{c} \pm x \omega_{\mathrm{RF}}, x \neq 1\right)$ employing DSBCS modulation scheme unavoidable, as shown in Figs. 2 and 5. These unwanted optical sidebands can degrade the performance of the desired electrical mm-wave signals. For mm-wave signal generation using balanced MZMs, the amplitudes of the optical sidebands are only proportional to the Bessel functions of the corresponding orders. However, for mm-wave signal generations using imbalanced MZMs, the amplitudes of optical sidebands are related to not only the Bessel functions of the corresponding orders but also the weighting factors consisting of $E_{\mathrm{AM}}$ and $E_{\mathrm{PM}}$ which are defined in Appendix II. As modulation index (MI $=V_{p-p} / 2 V_{\pi}, V_{p-p}$ is the peak-to-peak voltage of the MZM driving signal) for driving MZM increases from zero to one, the zeroth-order Bessel function and the Bessel function of 


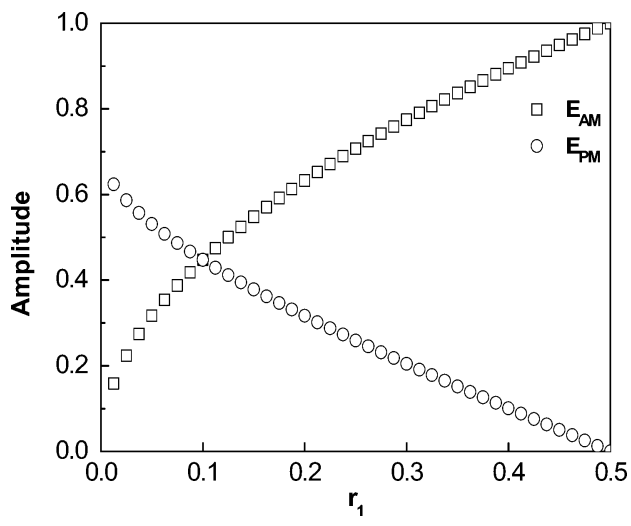

Fig. 7. $E_{\mathrm{AM}}$ and $E_{\mathrm{PM}}$ varied with $r_{1}$.

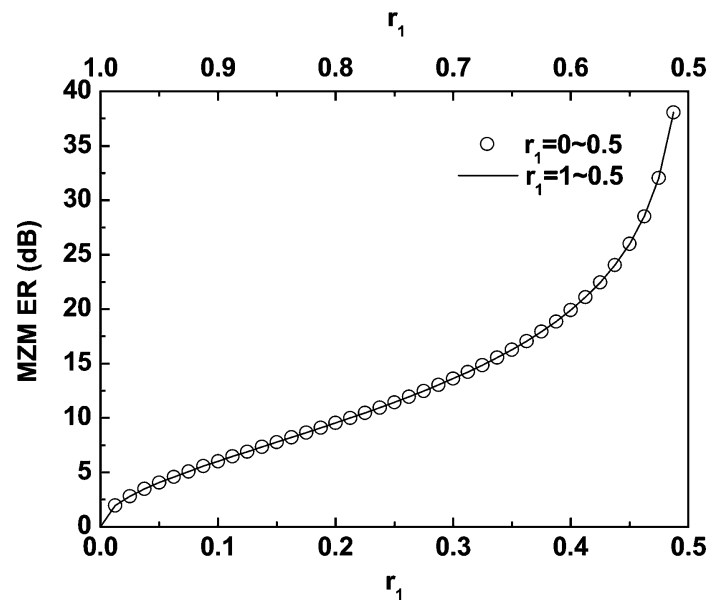

Fig. 8. MZM ER varied with $r_{1}$.

order of more than one will decrease and increase, respectively. Fig. 7 plots $E_{\mathrm{AM}}$ and $E_{\mathrm{PM}}$ as a function of $r_{1}$, with $r_{2}$ set at 0.5 . As $r_{1}$ moves away from the ideal condition, decreasing from 0.5 to zero, as expected, the pure amplitude modulation term $E_{\mathrm{AM}}$ declines and the phase modulation term $E_{\mathrm{PM}}$ increases. The ER of MZM is determined by the combined effect of $r_{1}$ and $r_{2}$. Fig. 8 plots the ER versus $r_{1}$, for a commercially available MZM, the typical value of ER is $25 \mathrm{~dB}$, which corresponds to $r_{1}=0.44$.

For the mm-wave signal produced by DSBCS modulation, the two first-order sidebands are the desired optical signals, and the suppression ratio of the other undesired distortion sideband caused by the MZM nonlinearity and the imbalance is the key parameter in the RoF system. The commercial software, VPI WDM-TransmissionMaker 5.0, is used to simulate numerically the suppression ratio. Fig. 9 shows the optical carrier and distortion suppression ratio (OCDSR, $P_{1 x}$ ) as a function of MI for the driving the MZM with an ER of $25 \mathrm{~dB}$. The $P_{1 x}$ is defined as

$$
P_{1 x}=\frac{P_{1}}{P_{x}}
$$

where $P_{1}$ and $P_{1 x}$ are the optical powers of the first-order sideband $\left(\omega_{c} \pm \omega_{\mathrm{RF}}\right)$ and the $x$ th-order sideband $\left(\omega_{c} \pm x \cdot \omega_{\mathrm{RF}}, x \neq\right.$ $1)$, respectively. Notably, $P_{10}$ is the OCSR. As MI falls from one to zero, the distortion suppression ratios $\left(P_{1 x}, x=2,3,4,5\right)$ are improved, but the OCSR $\left(P_{10}\right)$ decreases. When the MZM ER

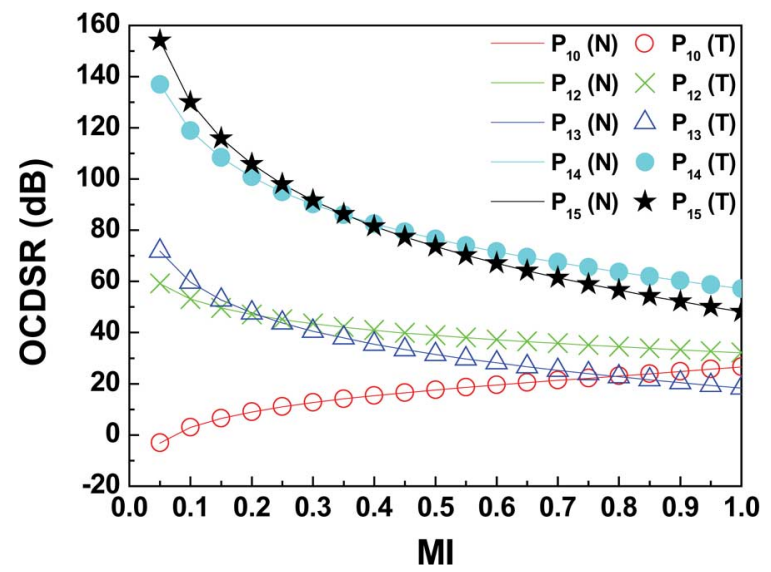

Fig. 9. OCDSR versus the MI for driving MZM. The MZM ER is $25 \mathrm{~dB}$. A: Theoretical analysis, N: Numerical stimulation.

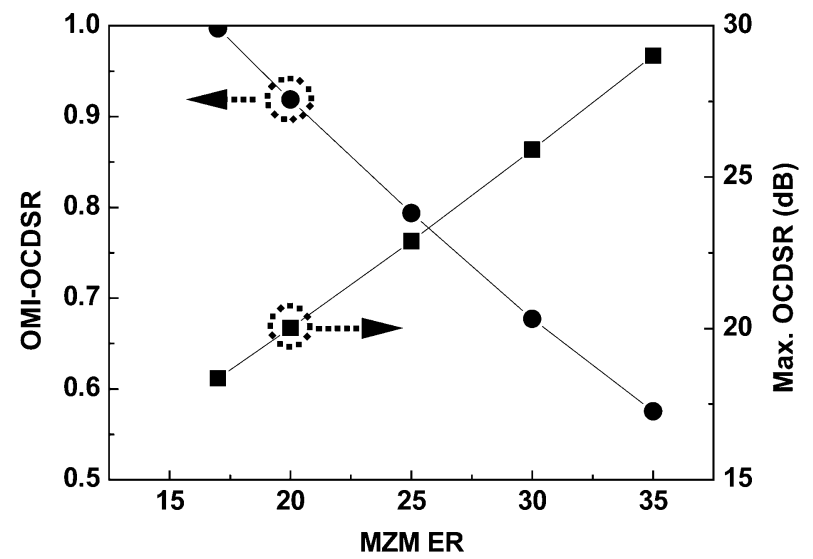

Fig. 10. OMI-OCDSR and the corresponding maximum OCDSR versus MZM ER.

equals $25 \mathrm{~dB}$ and MI exceeds 0.79 , the third-order optical sideband $\left(P_{13}\right)$ is key to determining the degradation of OCDSR. However, for $\mathrm{MI}<0.79, \mathrm{P}_{10}$ is less than $\mathrm{P}_{13}$, and the OCSR, rather than OCDSR, dominates the system performance. Therefore, the optimal MI for maximum OCDSR (OMI-OCDSR) exists at the intersection of $\mathrm{P}_{10}$ and $\mathrm{P}_{13}$ and is determined by the MZM ER. Fig. 10 plots the OMI-OCDSR and the corresponding maximum OCDSR versus the MZM ER. When the MZM ER is less than $17 \mathrm{~dB}, \mathrm{P}_{10}$ is always less than $\mathrm{P}_{13}$. Thus, the OCSR controls the system performance and the OMI-OCDSR is one. For a commercially available MZM with a typical ER of 25 $\mathrm{dB}$, the OMI-OCDSR is about 0.79 and the corresponding maximum OCDSR is $23 \mathrm{~dB}$.

\section{Generated Electrical mm-Wave Signal Analysis}

Fig. 6(b) presents the generated electrical spectra following square-law PD detection. The desired electrical signal originates from the beating between two optical sidebands with a frequency difference that is twice the frequency of the electrical driving signal, and the beating of the two first-order optical sidebands dominates the power intensity of the desired electrical signal. Notably, $E_{\mathrm{AM}} / E_{\mathrm{PM}}$ and $\mathrm{m}$, which originate in MZM imbalance and MI for driving MZM, respectively, determine the power intensity of the desired and undesired electrical signal, 


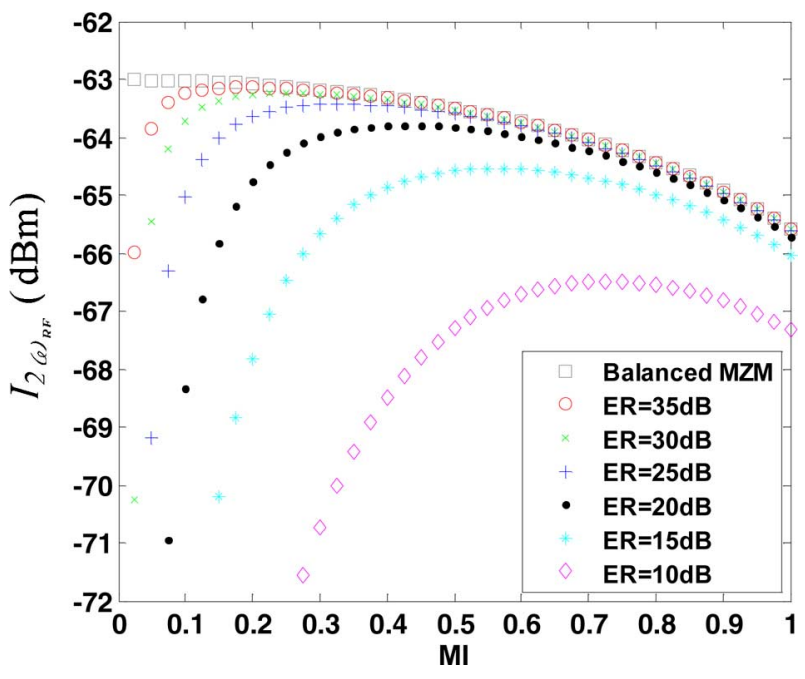

Fig. 11. $I_{2 \omega_{\mathrm{RF}}}$ versus MI and MZM ER after PD detectin.

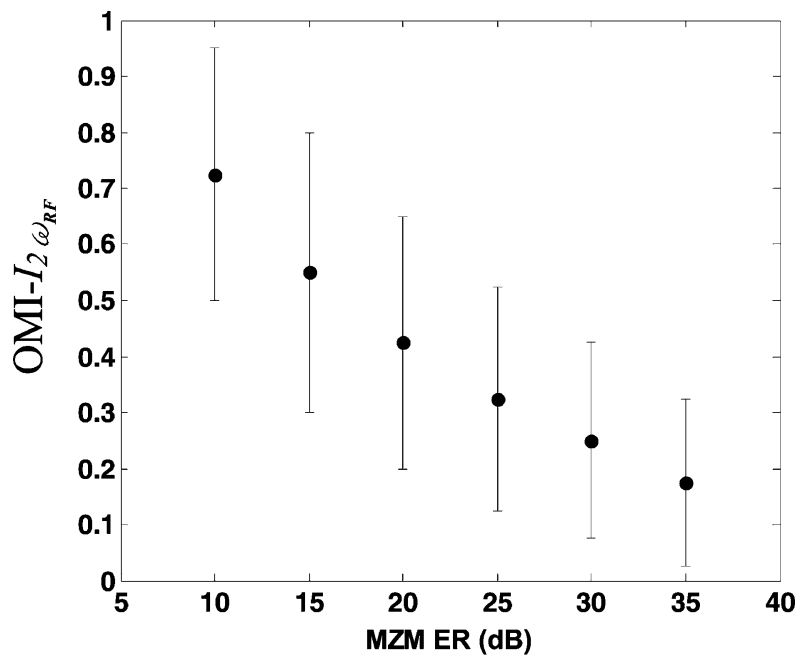

Fig. 12. OMI- $I_{2 \omega_{\mathrm{RF}}}$ versus MZM ER. The solid circle indicates the OMI- $I_{2 \omega_{\mathrm{RF}}}$, and the error-bar of the OMI- $I_{2 \omega_{\mathrm{RF}}}$ corresponds to $1-\mathrm{dB}$ tolerance of the maximum $I_{2 \omega_{\mathrm{RF}}}$.

as shown in Appendix II. For optical mm-wave signal generation or optical up-conversion for RoF links, since the undesired electrical signals can be easily removed by an electrical filter, the normalized power intensity of the desired electrical signal $\left(I_{2 \omega_{\mathrm{RF}}}\right)$ is the key parameter as the MZM ER and MI vary.

Fig. 11 plots the $I_{2 \omega_{\mathrm{RF}}}$ versus MI and MZM ER after PD detection. The power of the optical $\mathrm{mm}$-wave signals before PD detection is normalized to be $0 \mathrm{dBm}$. As MI increases from zero to one, the $I_{2 \omega_{\mathrm{RF}}}$ increases first and then declines. Notably, the maximum $I_{2 \omega_{\mathrm{RF}}}$ decreases as the MZM ER decreases. Furthermore, the optimal MI for the maximum $I_{2 \omega_{\mathrm{RF}}}\left(\mathrm{OMI}-I_{2 \omega_{\mathrm{RF}}}\right)$ increases as the MZM ER falls from 35 to $10 \mathrm{~dB}$, as shown in Fig. 12. For a commercial MZM with a typical ER of $25 \mathrm{~dB}$, the OMI- $I_{2 \omega_{\mathrm{RF}}}$ is $0.32 \pm 0.2$ for a maximum $I_{2 \omega_{\mathrm{RF}}}$ with a tolerance of $1 \mathrm{~dB}$.

Since fiber chromatic dispersion induces different phase variations of the optical sidebands, $I_{2 \omega_{\mathrm{RF}}}$ varies with the transmission length of the SSMF. Fig. 13(a) plots the power intensity fluctuation of the electrical $40-\mathrm{GHz} \mathrm{mm}$-wave signal that

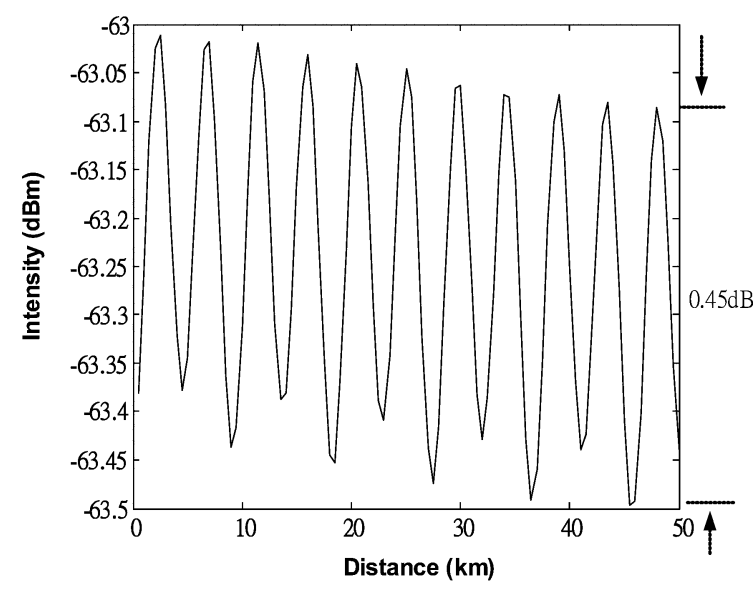

(a)

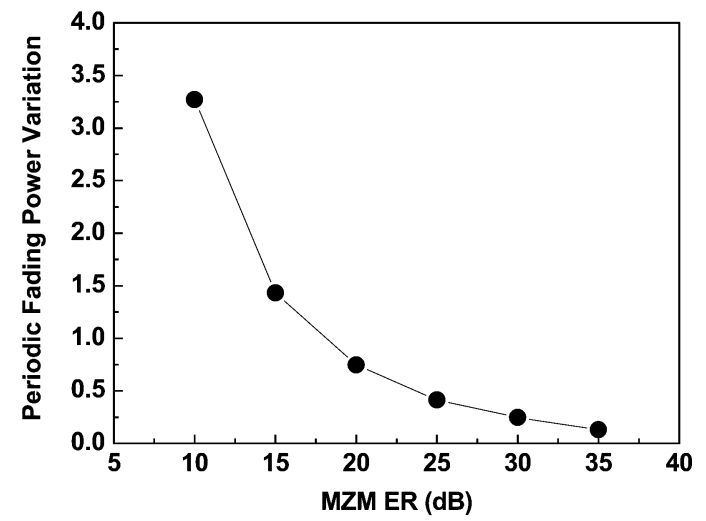

(b)

Fig. 13. (a) Power fluctuation of the 40-GHz mm-wave signal transmitted over 50-km SSMF. (b) Periodic fading power variation versus the MZM ER following 50-km transmission of SSMF.

is transmitted over $50-\mathrm{km}$ SSMF. The MZM ER is $25 \mathrm{~dB}$ and the power of the optical $\mathrm{mm}$-wave signal is normalized to be $0 \mathrm{dBm}$ before PD detection. The periodic fading power variation, which is associated with undesired optical sidebands, is less than $0.5 \mathrm{~dB}$. Fig. 13(b) plots the periodic fading power variation versus the MZM ER following 50-km transmission of SSMF. As the MZM ER falls, the periodic fading power variation increases. When the MZM ER is $10 \mathrm{~dB}$, the periodic fading power variation reaches $3.3 \mathrm{~dB}$ and the receiver sensitivity of the mm-wave signal is clearly degraded. When the MZM ER exceeds $20 \mathrm{~dB}$, the periodic fading power variation is less than $1 \mathrm{~dB}$ and is negligible.

\section{E. Comparison of Different Optimal MIs}

In summary, the MZM nonlinearity and imbalance were theoretically demonstrated to strongly influence the performance of the optical mm-wave signals based on the DSBCS modulation scheme. Table I presents the different optimal MIs for the maximum OCDSR and desired electrical signal $I_{2 \omega_{\mathrm{RF}}}$ for different applications. The optimal MI that maximizes OCDSR can be used to generate a tunable optical mm-wave signal for a wideband surveillance, spread spectrum or software-defined radio [12], [13]. For those systems, the undesired electrical signal 
TABLE I

COMPARISON OF DIFFERENT OPTIMAL MI FOR DRIVING MZ

\begin{tabular}{|l|l|l|}
\hline Optimal MI & \multicolumn{1}{|c|}{ Application } & Contribution \\
\hline OCDSR & Tunable optical mm-wave signal & 1.With MZM ER $>25 \mathrm{~dB}$, \\
& for wideband surveillance, spread & OMI-OCDSR $<0.79$ and \\
& spectrum, and software-defined & OCDSR $>23 \mathrm{~dB}$ \\
& radio. & 2.Signal improvement \\
\hline Frequency & 1.BroadBand wireless mm-wave & $\begin{array}{l}\text { 1.With MZM ER }>25 \mathrm{~dB}, \\
\text { OMI- } I_{2 \omega_{R F}}<0.32\end{array}$ \\
doubling & signal generation & 2.Signal improvement \\
Electrical & 2.Optical up-conversion & \\
Signals & & \\
\hline
\end{tabular}

cannot be filtered out after PD detection because the RF frequency is not fixed. Therefore, OMI-OCDSR can be used to achieve high undesired electrical distortion ratio. The optimal MI for maximum $I_{2 \omega_{\mathrm{RF}}}$ can applied to optical broadband wireless mm-wave signal generation or optical up-conversion in RoF access systems. In a conventional DSBCS modulation scheme, the DD-MZM is adopted to yield full MI $(\mathrm{MI}=1)$. According to the simulation results, only SD-MZM is required to realize DSBCS modulation, which provides a compact, cost-effective, and better performance alternative.

\section{OpticAl RF Signal GENERATION USING DSBCS MODULATION}

\section{A. Experimental Setup}

Fig. 14 shows the experimental setup used for optical RF signal generation and transmission based on DSBCS modulation scheme. The continuous wave $(\mathrm{CW})$ laser is generated by a distributed feedback laser, and the emission wavelength is $1540 \mathrm{~nm}$. The baseband signal is $622-\mathrm{Mb} / \mathrm{s}$ on-off-keying (OOK) pseudo random bit sequence (PRBS) signal with a word length of $2^{31}-1$ and up-converted using a 5-GHz sinusoidal signal, as shown in inset (i) of Fig. 14. The up-converted signal is amplified to maximum $V_{p-p}$ of $7 \mathrm{~V}$, which is limited by the available commercial RF amplifier. To realize DSBCS modulation, the MZM is biased at the minimum transmission point and DD-MZM with $V_{\pi}$ of $5 \mathrm{~V}$ is utilized to yield full MI $(\mathrm{MI}=1)$. The frequency of the generated optical microwave is $10 \mathrm{GHz}$. The insets (ii) and (iii) of Fig. 14 present the optical microwave waveform and spectrum, respectively. The generated optical signal is amplified using an erbium-doped fiber amplifier (EDFA) and then filtered through a tunable optical filter with a bandwidth of $0.4 \mathrm{~nm}$. After it is transmitted over SSMF, the optical microwave signal is converted into an electrical microwave signal using a PD with a $3-\mathrm{dB}$ bandwidth of $38 \mathrm{GHz}$, and the converted electrical signal is amplified by an electrical amplifier. The electrical microwave signal is down-converted by a mixer with a $10-\mathrm{GHz}$ sinusoidal signal and then passes through a low-pass filter with a 3-dB bandwidth of $622 \mathrm{MHz}$. The inset (iv) in Fig. 14 shows eye diagrams of the down-converted signals. The down-converted signals are

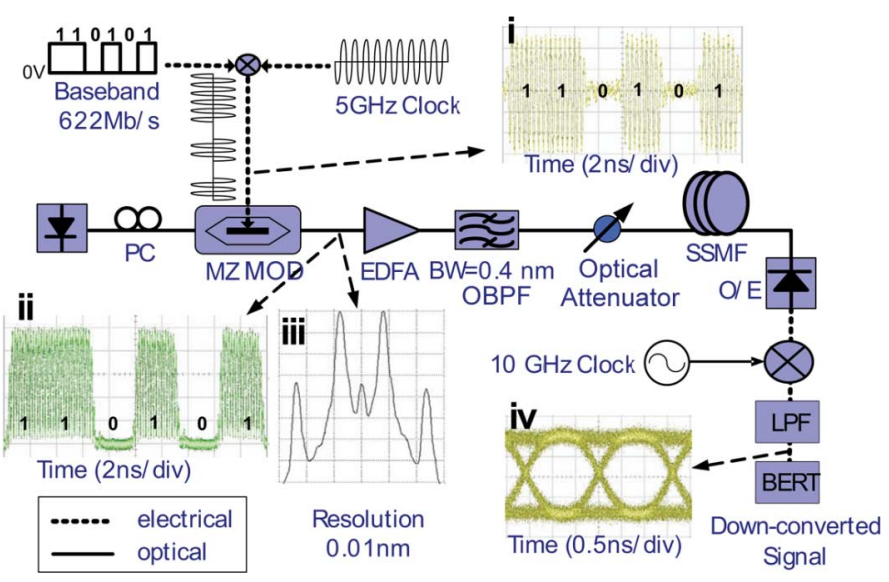

Fig. 14. Experimental setup for optical microwave generation based on DSBCS modulation scheme. (i) Up-converted electrical signal. (ii) Generated optical microwave signal. (iii) Optical spectrum of generated microwave signal. (iv) Eye diagram of down-converted signals.

tested by a bit-error-ratio (BER) tester. The fiber length is set to 25,50 , and $75 \mathrm{~km}$.

\section{B. Experimental Results and Discussions}

The performance of RF signals is strongly related to the MZM nonlinearity which can be reduced by decreasing the MI. Fig. 15(a) plots the variation of the receiver sensitivities of the down-converted signals as a function of MI. The receiver sensitivity initially increases and then decreases as MI falls from 1 to 0.13 , and the sensitivity is minimal at the optimal MI of 0.43 . As theoretically demonstrated above, the optimal MI is determined by the tradeoff between the MZM nonlinearity and the residual optical carrier caused by the imbalance y-junction splitting ratio of MZM.

The MZM nonlinearity and OCSR are determined by MI. As the MI that drives MZM decreases from 1 to 0.18 , the OCSR decrease from 17 to $4.5 \mathrm{~dB}$; meanwhile, the amplitude of the second-order optical sideband that is associated with the MZM nonlinearity decreases, as shown in Fig. 16. Note that the OCSRs in Fig. 9 are better than those in Fig. 16. The reason is that we use OOK PRBS data for experimental demonstration in Fig. 16, instead of a sinusoidal signal that is used for theoretical analysis in Fig. 9. Furthermore, the reduction of the MZM nonlinearity makes the duty cycle of optical microwaves closer to 0.5 as shown in Fig. 17. At a given optical power, a smaller duty cycle of the optical microwaves corresponds to a higher peak power, resulting in better receiver sensitivity of the down-converted signal. However, a low OCSR is such that the optical power of two first-order sidebands are relatively low and optical power of the residual carrier is relatively high, worsening the sensitivity of the receiver signal. Therefore, a tradeoff for the receiver sensitivity exists between the MZM nonlinearity and OCSR as MI is decreased.

When the optimal MI is 0.43 , the receiver sensitivity of the down-converted signals at a BER of $10^{-9}$ is $-22.7 \mathrm{dBm}$. The receiver sensitivity of the down-converted signal improves by 1 $\mathrm{dB}$ as the MI decreases from 1 to 0.43 . As $0.23<\mathrm{MI}<0.55$, the power penalty of down-converted signals, which are compared with those using optimal MI of 0.43 , is less than $0.2 \mathrm{~dB}$, 


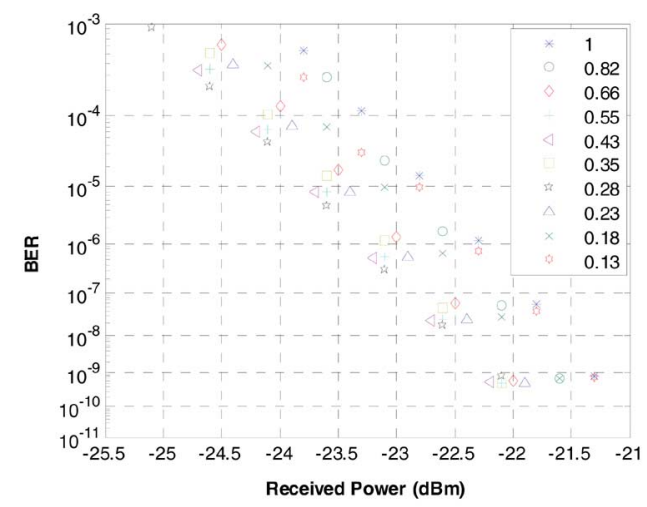

(a)

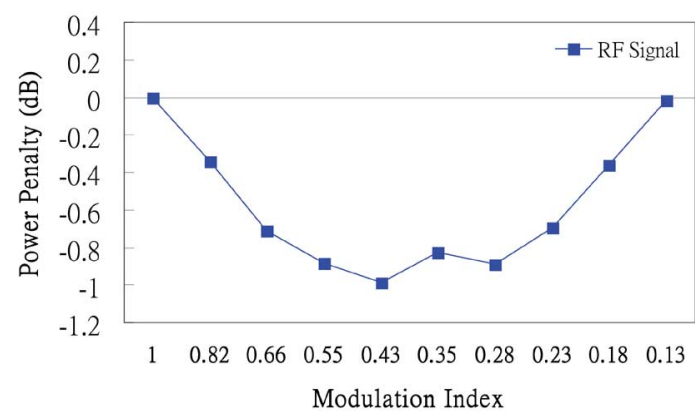

(b)

Fig. 15. BER curves (a) and power penalties at BER of $10^{-9}$ (b) of downconverted signals versus MI. The receiver sensitivity at MI $=1$ is used as a reference for the power penalty.

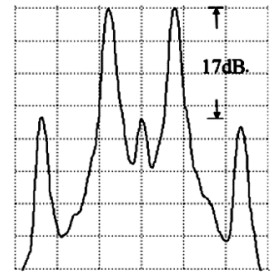

(a)

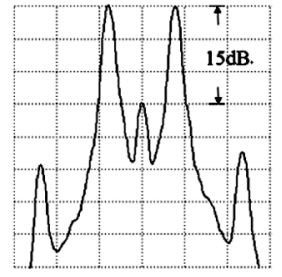

(b)

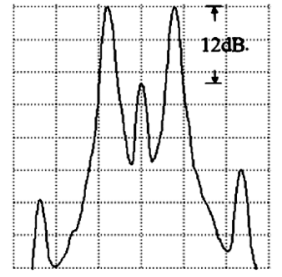

(c)

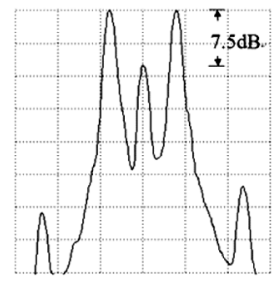

(d)

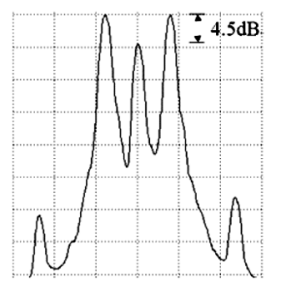

(e)
Fig. 16. OCSRs of optical microwave signals based on DSBCS modulation. The resolution is $0.01 \mathrm{~nm}$. The MI is set at (a) 1 , (b) 0.66 , (c) 0.43 , (d) 0.28 , and (e) 0.18 .

as shown in Fig. 15(b). The ER of the MZM used in the experiment is around $25 \mathrm{~dB}$, and the simulation results show that the OMI- $I_{2 \omega_{\mathrm{RF}}}$ is $0.32 \pm 0.2$ over a $1-\mathrm{dB}$ tolerance of the maximum $I_{2 \omega R F}$, as shown in Fig. 12. Therefore, the experimental result is well consistent with the simulated result. Since the optimal MI is 0.43 , the driving $V_{p-p}$ for commercially available MZM with typical $V_{\pi}$ of $5 \mathrm{~V}$ is $4.3 \mathrm{~V}\left(0.43 * 2 V_{\pi}\right)$. Hence, the DD-MZM can be replaced with SD-MZM. After the optical microwave signals using the optimal MI of 0.43 are transmitted over $25-\mathrm{km}, 50-\mathrm{km}$,

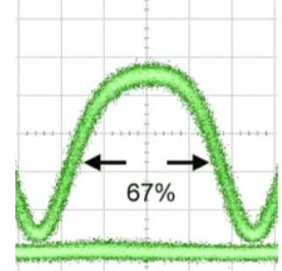

(a)

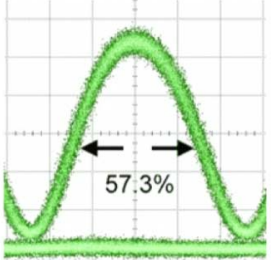

(b)

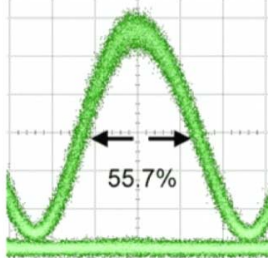

(c) (d)

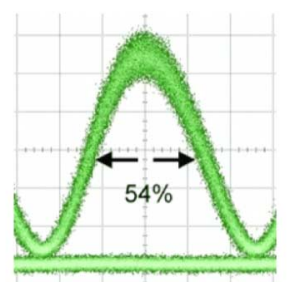

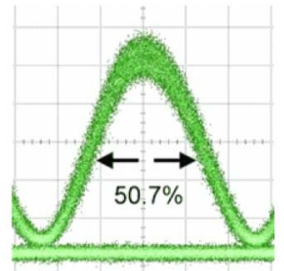

(e)
Fig. 17. Duty cycles of optical microwave signal based on DSBCS modulation. The optical microwave power is $1 \mathrm{dBm}$. The optical power scale is $0.8 \mathrm{~mW} / \mathrm{div}$ and the time scale is $20 \mathrm{ps} / \mathrm{div}$. The MI is set at (a) 1 , (b) 0.66 , (c) 0.43 , (d) 0.28 , and (e) 0.18

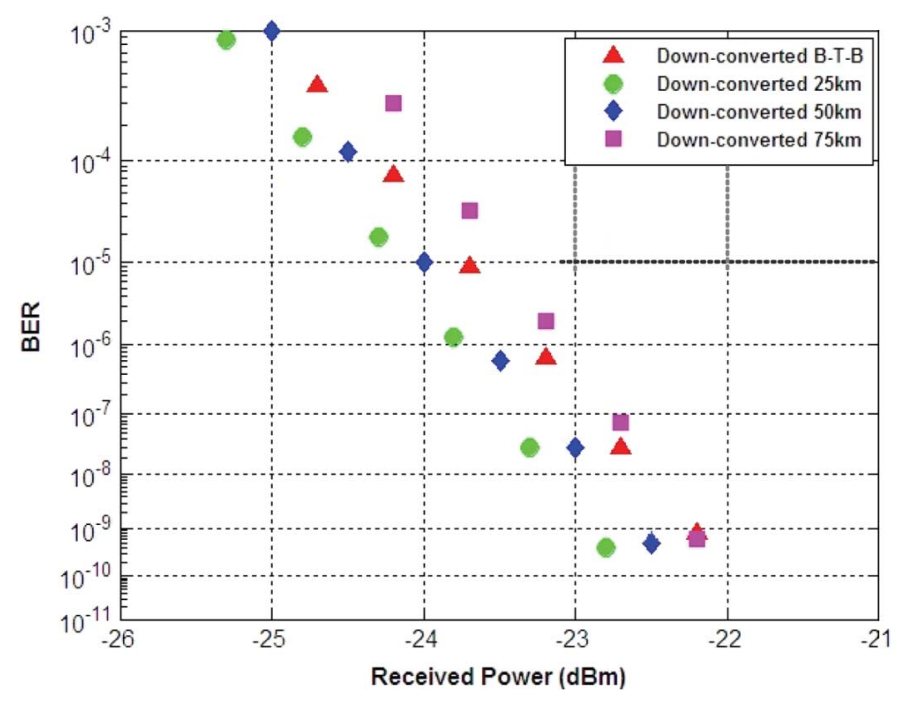

Fig. 18. BER curves of optical microwave signals using optimal MI of 0.43 after transmission over $25 \mathrm{~km}, 50 \mathrm{~km}$, and $75 \mathrm{~km} \mathrm{SSMF}$.

and 75-km SSMF, the power penalty for the receiver sensitivity of the down-converted signals at BER of $10^{-9}$ is less than $1 \mathrm{~dB}$ as shown in Fig. 18.

\section{CONCLUSION}

The impact of the inherent MZM nonlinear distortion on mm-wave signal generation by DSBCS modulation was theoretically and experimentally investigated. Given fabrication tolerances, the effect of MZM imbalance on mm-wave generation was theoretically and experimentally studied. Moreover, the optimum conditions for tunable optical mm-wave signal generation, optical up-conversion, and optical broadband wireless mm-wave signal generation using DSBCS modulation have been also discussed. According to the simulation results, only $\mathrm{SD}-\mathrm{MZM}$ is required to realize DSBCS modulation, which 
provides a compact, cost-effective and better performance alternative.

\section{APPENDIX I}

Expanding (1) with Bessel function leads to an expression for the electrical field at the output of the MZM as

$$
\begin{aligned}
E_{\text {out }}( & t) \\
= & E_{o} \cos b\left\{J_{o}(m) \cos \left(\omega_{c} t\right)\right. \\
& +\sum_{n=1}^{\infty}\left[J_{2 n}(m) \cos \left(\omega_{c} t+2 n \omega_{\mathrm{RF}} t-n \pi\right)\right. \\
& \left.\left.+J_{2 n}(m) \cos \left(\omega_{c} t-2 n \omega_{\mathrm{RF}} t+n \pi\right)\right]\right\} \\
& +E_{o} \sin \mathrm{b}\left\{\sum _ { n = 1 } ^ { \infty } \left[J _ { 2 n - 1 } ( m ) \operatorname { c o s } \left(\omega_{c} t\right.\right.\right. \\
& \left.+(2 n-1) \omega_{\mathrm{RF}} t-n \pi\right) \\
& \left.\left.+J_{2 n-1}(m) \cos \left(\omega_{c} t-(2 n-1) \omega_{\mathrm{RF}} t+n \pi\right)\right]\right\}
\end{aligned}
$$

where $J_{n}$ is the Bessel function of the first kind of order $\mathrm{n}$, the bias voltage is set at $V_{\text {bias }}=V_{\pi}$, and $\cos \mathrm{b}=0$ and $\sin \mathrm{b}=1$. Consequently, the electrical field of the mm-wave signal using DSBCS modulation can be written as

$$
\begin{aligned}
E_{\text {out }}(t)= & E_{o} \cdot \sum_{n=1}^{\infty}\left[J _ { 2 n } ( m ) \operatorname { c o s } \left(\omega_{c} t\right.\right. \\
& \left.+2 n \omega_{\mathrm{RF}} t-n \pi\right)+J_{2 n-1}(m) \cos \left(\omega_{c} t\right. \\
& \left.\left.\left.-(2 n-1) \omega_{\mathrm{RF}} t+n \pi\right)\right]\right\} .
\end{aligned}
$$

The amplitudes of the generated optical sidebands are proportional to those of the corresponding Bessel functions associated with the phase modulation index $\mathrm{m}$. With the amplitude of the electrical driving signal $V_{m}$ equal to $V_{\pi}$, the maximum $\mathrm{m}$ is $(\pi / 2)$. As $0<m<(\pi / 2)$, the Bessel function $J_{2 n}$ for $n \geq 1$ decreases and increases with the order of Bessel function and $\mathrm{m}$, respectively. $J_{1}((\pi / 2)), J_{3}((\pi / 2)), J_{5}((\pi / 2))$, and $J_{7}((\pi / 2))$ are $0.5668,0.0690,0.0022$, and 0.00003581 , respectively. Therefore, the optical sidebands with the Bessel function higher than $J_{5}(\mathrm{~m})$ can be ignored, and (A2) can be further simplified to

$$
\begin{aligned}
E_{\mathrm{out}}(t)= & E_{o}\left\{-J_{1}(m) \cos \left[\left(\omega_{c}+\omega_{\mathrm{RF}}\right) t\right]\right. \\
& -J_{1}(m) \cos \left[\left(\omega_{c}-\omega_{\mathrm{RF}}\right) t\right] \\
& +J_{3}(m) \cos \left[\left(\omega_{c}+3 \omega_{\mathrm{RF}}\right) t\right] \\
& +J_{3}(m) \cos \left[\left(\omega_{c}-3 \omega_{\mathrm{RF}}\right) t\right] \\
& -J_{5}(m) \cos \left[\left(\omega_{c}+5 \omega_{\mathrm{RF}}\right) t\right] \\
& \left.-J_{5}(m) \cos \left[\left(\omega_{c}-5 \omega_{\mathrm{RF}}\right) t\right]\right\} .
\end{aligned}
$$

When optical DSBCS signals are transmitted over a singlemode fiber with dispersion, a phase shift to each optical side- band relative to optical carrier is induced. The propagation constant of the dispersion fiber $\beta(\omega)$ can be expressed as

$$
\begin{aligned}
\beta(\omega) & =n(\omega) \frac{\omega}{c} \\
& =\beta_{o}+\beta_{1}\left(\omega-\omega_{c}\right)+\frac{1}{2} \beta_{2}\left(\omega-\omega_{c}\right)^{2}+\cdots
\end{aligned}
$$

where $\beta_{m}=\left.\left(d^{m} \beta / d \omega^{m}\right)\right|_{\omega=\omega_{c}}$ is the derivative of the propagation constant evaluated at $\omega=\omega_{c}$. The effect of high-order fiber dispersion at $1550-\mathrm{nm}$ band is neglected. For carrier tones with central frequency at $\omega=\omega_{c} \pm n \omega_{\mathrm{RF}}$, we have

$$
\beta\left(\omega_{c} \pm n \omega_{\mathrm{RF}}\right) \cong \beta_{o} \pm n \beta_{1}\left(\omega_{c}\right) \omega_{\mathrm{RF}}+\frac{1}{2} n^{2} \beta_{2}\left(\omega_{c}\right) \omega_{\mathrm{RF}}^{2}
$$

and

$$
\beta_{2}\left(\omega_{c}\right)=-\frac{c}{2 \pi f_{c}^{2}} \cdot D\left(\omega_{c}\right)
$$

where $c$ is light speed in free space, $D$ is the chromatic dispersion parameter, and $f_{c}$ is the frequency of the optical carrier. For a standard single-mode fiber, $D$ is $17-\mathrm{ps} /(\mathrm{nm} . \mathrm{km})$. The fiber loss is ignored. Therefore, after transmission over a single-mode fiber of length $z$, the electrical field can be written as

$$
\begin{aligned}
E(t)= & E_{o}\left\{-J_{1}(m) \cos \left[\left(\omega_{c}+\omega_{\mathrm{RF}}\right) t\right.\right. \\
& \left.-\beta_{o} z-\beta_{1} \omega_{\mathrm{RF}} z-\frac{1}{2} \beta_{2} \omega_{\mathrm{RF}}^{2} z\right] \\
& -J_{1}(m) \cos \left[\left(\omega_{c}-\omega_{\mathrm{RF}}\right) t\right. \\
& \left.-\beta_{o} z+\beta_{1} \omega_{\mathrm{RF}} z-\frac{1}{2} \beta_{2} \omega_{\mathrm{RF}}^{2} z\right] \\
& +J_{3}(m) \cos \left[\left(\omega_{c}+3 \omega_{\mathrm{RF}}\right) t\right. \\
& \left.-\beta_{o} z-3 \beta_{1} \omega_{\mathrm{RF}} z-\frac{9}{2} \beta_{2} \omega_{\mathrm{RF}}^{2} z\right] \\
& +J_{3}(m) \cos \left[\left(\omega_{c}-3 \omega_{\mathrm{RF}}\right) t\right. \\
& \left.-\beta_{o} z+3 \beta_{1} \omega_{\mathrm{RF}} z-\frac{9}{2} \beta_{2} \omega_{\mathrm{RF}}^{2} z\right] \\
& \left.\left.-\beta_{o} z+5 \beta_{1} \omega_{\mathrm{RF}} z-\frac{25}{2} \beta_{2} \omega_{\mathrm{RF}}^{2} z\right]\right\} \\
& -J_{5}(m) \cos \left[\left(\omega_{c}+5 \omega_{\mathrm{RF}}\right) t\right. \\
& \left.-\beta_{o} z-5 \beta_{1} \omega_{\mathrm{RF}} z-\frac{25}{2} \beta_{2} \omega_{\mathrm{RF}}^{2} z\right] \cos \left[\left(\omega_{c}-5 \omega_{\mathrm{RF}}\right) t\right. \\
& \\
& \\
& \\
&
\end{aligned}
$$

After square-law detection using an ideal PD with responsitivity $\mathrm{R}$, the photocurrent can be expressed as

$$
i(t)=R \cdot|E(t)|^{2} \text {. }
$$

The individual square term of (A8) will generate the baseband signal, and the cross terms will generate the desired 
mm-wave signal and its harmonics distortion signals. These desired mm-wave terms can be expressed as

$$
\begin{aligned}
i_{2 \omega_{\mathrm{RF}}}= & R \cdot E_{o}^{2}\left[J_{1}^{2}(m)-2 \sum_{n=1}^{\infty} J_{2 n-1}(m)\right. \\
& \left.\cdot J_{2 n+1}(m) \cos \left(4 n \beta_{2} \omega_{\mathrm{RF}}^{2} z\right)\right] \\
& \cdot \cos \left(2 \omega_{\mathrm{RF}} t-2 \beta_{1} \omega_{\mathrm{RF}} z\right) \\
i_{4 \omega_{\mathrm{RF}}}= & R \cdot E_{o}^{2}\left[-2 J_{1}(m) J_{3}(m) \cos \left(4 \beta_{2} \omega_{\mathrm{RF}}^{2} z\right)\right. \\
+ & \left.2 J_{1}(m) J_{5}(m) \cdot \cos \left(12 \beta_{2} \omega_{\mathrm{RF}}^{2} z\right)\right] \\
& \cdot \cos \left(4 \omega_{\mathrm{RF}} t-4 \beta_{1} \omega_{\mathrm{RF}} z\right) \\
i_{6 \omega_{\mathrm{RF}}}= & R \cdot E_{o}^{2}\left[J_{3}^{2}(m)+2 J_{1}(m) J_{5}(m) \cos \left(12 \beta_{2} \omega_{\mathrm{RF}}^{2} z\right)\right] \\
& \cdot \cos \left(6 \omega_{\mathrm{RF}} t-6 \beta_{1} \omega_{\mathrm{RF}} z\right) \\
i_{8 \omega_{\mathrm{RF}}=} & R \cdot E_{o}^{2}\left[-2 J_{3}(m) J_{5}(m) \cos \left(8 \beta_{2} \omega_{\mathrm{RF}}^{2} z\right)\right] \\
& \cdot \cos \left(8 \omega_{\mathrm{RF}} t-8 \beta_{1} \omega_{\mathrm{RF}} z\right) \\
i_{10 \omega_{\mathrm{RF}}=} & R \cdot E_{o}^{2} \cdot J_{5}^{2}(m) \cdot \cos \left(10 \omega_{\mathrm{RF}} t-10 \beta_{1} \omega_{\mathrm{RF}} z\right) . \quad(\mathrm{A} 9)
\end{aligned}
$$

\section{APPENDIX II}

Rearranged (3), the electrical fields of the balanced MZM and PM modulations, respectively, can be rewritten as

$$
\begin{aligned}
E_{\text {out_AM }}(t)= & \Re e\left\{E_{\mathrm{AM}} \cdot \cos \left(\frac{\varphi_{1}-\varphi_{2}}{2}\right)\right. \\
& \cdot \exp ^{\left.j\left(\frac{\varphi_{1}+\varphi_{2}}{2}\right) \cdot \exp \left(j \omega_{c} t\right)\right\}} \\
\text { and } & \\
E_{\text {out_PM }}(t)= & \Re e\left\{E_{\mathrm{PM}} \cdot \exp \left(j \varphi_{2}\right) \cdot \exp \left(j \omega_{c} t\right)\right\}
\end{aligned}
$$

where $E_{\mathrm{AM}}$ and $E_{\mathrm{PM}}$ are $2 E_{o} \sqrt{r_{1}} \cdot \sqrt{r_{2}}$ and $E_{o}\left(\sqrt{1-r_{1}}\right.$. $\left.\sqrt{1-r_{2}}-\sqrt{r_{1}} \cdot \sqrt{r_{2}}\right)$, respectively. For a single-arm MZM, $\varphi_{1}=-\varphi_{2}=-(\theta / 2),(\theta / 2)$ can be expressed as

$$
\frac{\theta}{2}=\frac{V_{\text {bias }}}{2 V_{\pi}} \pi+\frac{V_{m} \cos \left(\omega_{\mathrm{RF}} t\right)}{2 V_{\pi}} \pi \stackrel{\text { def }}{=} \mathrm{b}+\mathrm{m} \cos \left(\omega_{\mathrm{RF}} t\right)
$$

where $\mathrm{b}$ and $\mathrm{m}$ are $\left(V_{\mathrm{bias}} / 2 V_{\pi}\right) \pi$ and $\left(V_{m} / 2 V_{\pi}\right) \pi$, respectively. The output electrical field of the amplitude modulation can be rewritten as

$$
\begin{aligned}
E_{\text {out_AM }}(t) & E_{\mathrm{AM}}\left\{\operatorname { c o s } b \left[J_{o}(m) \cdot \cos \left(\omega_{c} t\right)\right.\right. \\
& +\sum_{n=1}^{\infty}\left[J_{2 n}(m) \cdot \cos \left(\omega_{c} t+2 n \omega_{\mathrm{RF}} t-n \pi\right)\right. \\
& \left.\left.+J_{2 n}(m) \cos \left(\omega_{c} t-2 n \omega_{\mathrm{RF}} t+n \pi\right)\right]\right] \\
& +\sin b\left[\sum _ { n = 1 } ^ { \infty } \left[J_{2 n-1}(m) \cos \left(\omega_{c} t+(2 n-1) \omega_{\mathrm{RF}} t-n \pi\right)\right.\right. \\
& \left.\left.+J_{2 n-1}(m) \cos \left(\omega_{c} t-(2 n-1) \omega_{\mathrm{RF}} t+n \pi\right)\right]\right\} .
\end{aligned}
$$

For the phase modulation, the output electrical field can be written as

$$
\begin{aligned}
E_{\text {out_PM }}(t)=E_{\mathrm{PM}} & \cdot \sum_{n=-\infty}^{\infty} J_{n}(m) \\
& \cdot \cos \left\{\left(\omega_{c}+n \omega_{\mathrm{RF}}\right) t+\left(b+\frac{n \pi}{2}\right)\right\} .
\end{aligned}
$$

For mm-wave signals based on DSBCS modulation with the bias voltage set at $V_{\text {bias }}=V_{\pi}$, we have $b=(\pi / 2), \cos b=0$, and $\sin b=1$. The output electrical filed of the amplitude and phase modulations can be rewritten as

$$
\begin{aligned}
E_{\text {out_AM }}(t)= & E_{\mathrm{AM}} \sum_{n=1}^{\infty}\left\{J_{2 n-1}(m)\right. \\
& \cdot \cos \left[\omega_{c} t+(2 n-1) \omega_{\mathrm{RF}} t-n \pi\right] \\
& \left.+J_{2 n-1}(m) \cos \left[\omega_{c} t-(2 n-1) \omega_{\mathrm{RF}} t+n \pi\right]\right\}
\end{aligned}
$$

and

$$
\begin{aligned}
E_{\text {out_PM }}(t)=E_{\mathrm{PM}} & \cdot \sum_{n=-\infty}^{\infty} J_{n}(m) \\
& \cdot \cos \left\{\left(\omega_{c}+n \omega_{\mathrm{RF}}\right) t+\left(\frac{n+1}{2}\right) \pi\right\} .
\end{aligned}
$$

It is reasonable that optical sidebands with the Bessel functions with the order higher than 5 can be ignored, and the electrical field at the output of the MZM with imbalanced power splitting ratios can be simplified to

$$
\begin{aligned}
E_{\mathrm{out}}(t)= & E_{\mathrm{out} \_\mathrm{AM}}(t)+E_{\mathrm{out} \_\mathrm{PM}}(t) \\
= & -E_{\mathrm{PM}} J_{o}(m) \sin \left(\omega_{c} t\right) \\
& -\left(E_{\mathrm{AM}}+E_{\mathrm{PM}}\right) J_{1}(m) \cos \left(\omega_{c} t+\omega_{\mathrm{RF}} t\right) \\
& -\left(E_{\mathrm{AM}}+E_{\mathrm{PM}}\right) J_{1}(m) \cos \left(\omega_{c} t-\omega_{\mathrm{RF}} t\right) \\
& +E_{\mathrm{PM}} J_{2}(m) \sin \left(\omega_{c} t+2 \omega_{\mathrm{RF}} t\right) \\
& +E_{\mathrm{PM}} J_{2}(m) \sin \left(\omega_{c} t-2 \omega_{\mathrm{RF}} t\right) \\
& +\left(E_{\mathrm{AM}}+E_{\mathrm{PM}}\right) J_{3}(m) \cos \left(\omega_{c} t+3 \omega_{\mathrm{RF}} t\right) \\
& +\left(E_{\mathrm{AM}}+E_{\mathrm{PM}}\right) J_{3}(m) \cos \left(\omega_{c} t-3 \omega_{\mathrm{RF}} t\right) \\
& -E_{\mathrm{PM}} J_{4}(m) \sin \left(\omega_{c} t+4 \omega_{\mathrm{RF}} t\right) \\
& -E_{\mathrm{PM}} J_{4}(m) \sin \left(\omega_{c} t-4 \omega_{\mathrm{RF}} t\right) \\
& -\left(E_{\mathrm{AM}}+E_{\mathrm{PM}}\right) J_{5}(m) \cos \left(\omega_{c} t+5 \omega_{\mathrm{RF}} t\right) \\
& -\left(E_{\mathrm{AM}}+E_{\mathrm{PM}}\right) J_{5}(m) \cos \left(\omega_{c} t-5 \omega_{\mathrm{RF}} t\right) .
\end{aligned}
$$


After transmission over dispersive fiber, the electric field can be written as

$$
\begin{aligned}
E(t)= & -E_{\mathrm{PM}} J_{o}(m) \sin \left(\omega_{c} t\right) \\
& -\left(E_{\mathrm{AM}}+E_{\mathrm{PM}}\right) J_{1}(m) \cos \left(\omega_{c} t+\omega_{\mathrm{RF}} t\right. \\
& \left.-\beta_{o} z-\beta_{1} \omega_{\mathrm{RF}} z-1 / 2 \beta_{2} \omega_{\mathrm{RF}}^{2}\right) \\
& -\left(E_{\mathrm{AM}}+E_{\mathrm{PM}}\right) J_{1}(m) \cos \left(\omega_{c} t-\omega_{\mathrm{RF}} t\right. \\
& \left.-\beta_{o} z+\beta_{1} \omega_{\mathrm{RF}} z-1 / 2 \beta_{2} \omega_{\mathrm{RF}}^{2}\right) \\
& +E_{\mathrm{PM}} J_{2}(m) \sin \left(\omega_{c} t+2 \omega_{\mathrm{RF}} t\right. \\
& \left.-\beta_{o} z-2 \beta_{1} \omega_{\mathrm{RF}} z-2 \beta_{2} \omega_{\mathrm{RF}}^{2}\right) \\
& +E_{\mathrm{PM}} J_{2}(m) \sin \left(\omega_{c} t-2 \omega_{\mathrm{RF}} t\right. \\
& \left.-\beta_{o} z+2 \beta_{1} \omega_{\mathrm{RF}} z-2 \beta_{2} \omega_{\mathrm{RF}}^{2}\right) \\
& +\left(E_{\mathrm{AM}}+E_{\mathrm{PM}}\right) J_{3}(m) \cos \left(\omega_{c} t+3 \omega_{\mathrm{RF}} t\right. \\
& \left.-\beta_{o} z-3 \beta_{1} \omega_{\mathrm{RF}} z-9 / 2 \beta_{2} \omega_{\mathrm{RF}}^{2}\right) \\
& +\left(E_{\mathrm{AM}}+E_{\mathrm{PM}}\right) J_{3}(m) \cos \left(\omega_{c} t-3 \omega_{\mathrm{RF}} t\right. \\
& \left.-\beta_{o} z+3 \beta_{1} \omega_{\mathrm{RF}} z-9 / 2 \beta_{2} \omega_{\mathrm{RF}}^{2}\right) \\
& -E_{\mathrm{PM}} J_{4}(m) \sin \left(\omega_{c} t+4 \omega_{\mathrm{RF}} t\right. \\
& \left.-\beta_{o} z-4 \beta_{1} \omega_{\mathrm{RF}} z-8 \beta_{2} \omega_{\mathrm{RF}}^{2}\right) \\
& -E_{\mathrm{PM}} J_{4}(m) \sin \left(\omega_{c} t-4 \omega_{\mathrm{RF}} t\right. \\
& \left.-\beta_{o} z+4 \beta_{1} \omega_{\mathrm{RF}} z-8 \beta_{2} \omega_{\mathrm{RF}}^{2}\right) \\
& -\left(E_{\mathrm{AM}}+E_{\mathrm{PM}}\right) J_{5}(m) \cos \left(\omega_{c} t+5 \omega_{\mathrm{RF}} t\right. \\
& \left.-\beta_{o} z-5 \beta_{1} \omega_{\mathrm{RF}} z-25 / 2 \beta_{2} \omega_{\mathrm{RF}}^{2}\right) \\
& -\left(E_{\mathrm{AM}}+E_{\mathrm{PM}}\right) J_{5}(m) \cos \left(\omega_{c} t-5 \omega_{\mathrm{RF}} t\right. \\
& \left.-\beta_{o} z+5 \beta_{1} \omega_{\mathrm{RF}} z-25 / 2 \beta_{2} \omega_{\mathrm{RF}}^{2}\right) .
\end{aligned}
$$

After square-law detection using a PD with responsitivity R, the photocurrents of the desired mm-wave signal and its harmonics distortion signals can be expressed as

$$
\begin{aligned}
& i_{\omega_{\mathrm{RF}}}=R \cdot E_{o}^{2}\left\{2\left(E_{\mathrm{AM}} E_{\mathrm{PM}}+E_{\mathrm{PM}}^{2}\right)\right. \\
& \cdot\left[J_{o}(m) J_{1}(m) \sin \left(\frac{1}{2} \beta_{2} \omega_{\mathrm{RF}}^{2} z\right)\right. \\
& +J_{1}(m) J_{2}(m) \sin \left(\frac{3}{2} \beta_{2} \omega_{\mathrm{RF}}^{2} z\right) \\
& +J_{2}(m) J_{3}(m) \sin \left(\frac{5}{2} \beta_{2} \omega_{\mathrm{RF}}^{2} z\right) \\
& +J_{3}(m) J_{4}(m) \sin \left(\frac{7}{2} \beta_{2} \omega_{\mathrm{RF}}^{2} z\right) \\
& \left.\left.+J_{4}(m) J_{5}(m) \sin \left(\frac{9}{2} \beta_{2} \omega_{\mathrm{RF}}^{2} z\right)\right]\right\} \\
& \cdot \cos \left(\omega_{\mathrm{RF}} t-\beta_{1} \omega_{\mathrm{RF}} z\right) \\
& i_{2 \omega_{\mathrm{RF}}}=R \cdot E_{o}^{2}\left\{\left(E_{\mathrm{AM}}+E_{\mathrm{PM}}\right)^{2} J_{1}^{2}(m)\right. \\
& -2 E_{\mathrm{PM}}^{2}\left[J_{o}(m) J_{2}(m) \cdot \cos \left(2 \beta_{2} \omega_{\mathrm{RF}}^{2} z\right)\right. \\
& \left.+J_{2}(m) J_{4}(m) \cos \left(6 \beta_{2} \omega_{\mathrm{RF}}^{2} z\right)\right] \\
& -2\left(E_{\mathrm{AM}}+E_{\mathrm{PM}}\right)^{2}\left[J_{1}(m) J_{3}(m) \cos \left(4 \beta_{2} \omega_{\mathrm{RF}}^{2} z\right)\right. \\
& \left.\left.+J_{3}(m) J_{5}(m) \cos \left(8 \beta_{2} \omega_{\mathrm{RF}}^{2} z\right)\right]\right\} \\
& \text { - } \cos \left(\omega_{\mathrm{RF}} t-2 \beta_{1} \omega_{\mathrm{RF}} z\right) \\
& i_{3 \omega_{\mathrm{RF}}}=R \cdot E_{o}^{2}\left\{2\left(E_{\mathrm{AM}} E_{\mathrm{PM}}+E_{\mathrm{PM}}^{2}\right)\right.
\end{aligned}
$$

$$
\begin{aligned}
& \cdot\left[-J_{o}(m) J_{3}(m) \sin \left(\frac{9}{2} \beta_{2} \omega_{\mathrm{RF}}^{2} z\right)\right. \\
& -J_{1}(m) J_{4}(m) \sin \left(\frac{15}{2} \beta_{2} \omega_{\mathrm{RF}}^{2} z\right) \\
& -J_{2}(m) J_{5}(m) \sin \left(\frac{21}{2} \beta_{2} \omega_{\mathrm{RF}}^{2} z\right) \\
& \left.\left.+J_{1}(m) J_{2}(m) \sin \left(\frac{3}{2} \beta_{2} \omega_{\mathrm{RF}}^{2} z\right)\right]\right\} \\
& \text { - } \cos \left(3 \omega_{\mathrm{RF}} t-3 \beta_{1} \omega_{\mathrm{RF}} z\right) \\
& i_{4 \omega_{\mathrm{RF}}}=R \cdot E_{o}^{2}\left\{2 E_{\mathrm{PM}}^{2} J_{0}(m) J_{4}(m) \cos \left(8 \beta_{2} \omega_{\mathrm{RF}}^{2} z\right)\right. \\
& +2\left(E_{\mathrm{AM}}+E_{\mathrm{PM}}\right)^{2} \cdot\left[J_{1}(m) J_{5}(m) \cos \left(12 \beta_{2} \omega_{\mathrm{RF}}^{2} z\right)\right. \\
& \left.-J_{1}(m) J_{3}(m) \cos \left(4 \beta_{2} \omega_{\mathrm{RF}}^{2} z\right)\right] \\
& \left.+E_{\mathrm{PM}}^{2} J_{2}(m) J_{2}(m)\right\} \cdot \cos \left(4 \omega_{\mathrm{RF}} t-4 \beta_{1} \omega_{\mathrm{RF}} z\right) \\
& i_{5 \omega_{\mathrm{RF}}}=R \cdot E_{o}^{2}\left\{2\left(E_{\mathrm{AM}} E_{\mathrm{PM}}+E_{\mathrm{PM}}^{2}\right)\right. \\
& \cdot\left[J_{o}(m) J_{5}(m) \sin \left(\frac{25}{2} \beta_{2} \omega_{\mathrm{RF}}^{2} z\right)\right. \\
& \text { - } J_{1}(m) J_{4}(m) \sin \left(\frac{15}{2} \beta_{2} \omega_{\mathrm{RF}}^{2} z\right) \\
& \left.\left.+J_{2}(m) J_{3}(m) \sin \left(\frac{5}{2} \beta_{2} \omega_{\mathrm{RF}}^{2} z\right)\right]\right\} \\
& \cdot \cos \left(5 \omega_{\mathrm{RF}} t-5 \beta_{1} \omega_{\mathrm{RF}} z\right) \\
& i_{6 \omega_{\mathrm{RF}}}=R \cdot E_{o}^{2}\left\{2\left(E_{\mathrm{AM}}+E_{\mathrm{PM}}\right)^{2}\right. \\
& \text { - } J_{1}(m) J_{5}(m) \cos \left(12 \beta_{2} \omega_{\mathrm{RF}}^{2} z\right)-2 E_{\mathrm{PM}}^{2} \\
& \text { - } J_{2}(m) J_{4}(m) \cos \left(6 \beta_{2} \omega_{\mathrm{RF}}^{2} z\right)+\left(E_{\mathrm{AM}}+E_{\mathrm{PM}}\right)^{2} \\
& \text {. } \left.J_{3}(m) J_{3}(m)\right\} \cdot \cos \left(6 \omega_{\mathrm{RF}} t-6 \beta_{1} \omega_{\mathrm{RF}} z\right) \\
& i_{7 \omega_{\mathrm{RF}}}=R \cdot E_{o}^{2}\left\{2\left(E_{\mathrm{AM}}+E_{\mathrm{PM}}\right)^{2}\right. \\
& \cdot\left[-J_{2}(m) J_{5}(m) \sin \left(\frac{21}{2} \beta_{2} \omega_{\mathrm{RF}}^{2} z\right)\right. \\
& \left.\left.+J_{3}(m) J_{4}(m) \sin \left(\frac{7}{2} \beta_{2} \omega_{\mathrm{RF}}^{2} z\right)\right]\right\} \\
& \cdot \cos \left(7 \omega_{\mathrm{RF}} t-7 \beta_{1} \omega_{\mathrm{RF}} z\right) \\
& i_{8 \omega_{\mathrm{RF}}}=R \cdot E_{o}^{2}\left\{E_{\mathrm{PM}}^{2} J_{4}(m) J_{4}(m)\right. \\
& -2\left(E_{\mathrm{AM}}+E_{\mathrm{PM}}\right)^{2} J_{3}(m) J_{5}(m) \\
& \left.\cdot \cos \left(8 \beta_{2} \omega_{\mathrm{RF}}^{2} z\right)\right\} \cdot \cos \left(8 \omega_{\mathrm{RF}} t-8 \beta_{1} \omega_{\mathrm{RF}} z\right) \\
& i_{9 \omega_{\mathrm{RF}}}=R \cdot E_{o}^{2}\left\{2\left(E_{\mathrm{AM}} E_{\mathrm{PM}}+E_{\mathrm{PM}}^{2}\right) J_{4}(m)\right. \\
& \text { - } \left.J_{5}(m) \sin \left(\frac{9}{2} \beta_{2} \omega_{\mathrm{RF}}^{2} z\right)\right\} \cdot \cos \left(9 \omega_{\mathrm{RF}} t-9 \beta_{1} \omega_{\mathrm{RF}} z\right) \\
& i_{10 \omega_{\mathrm{RF}}}=R \cdot E_{o}^{2}\left\{\left(E_{\mathrm{AM}}+E_{\mathrm{PM}}\right)^{2} J_{5}(m) J_{5}(m)\right\} \\
& \cdot \cos \left(10 \omega_{\mathrm{RF}} t-10 \beta_{1} \omega_{\mathrm{RF}} z\right) \text {. }
\end{aligned}
$$

\section{REFERENCES}

[1] T. Kamisaka, T. Kuri, and K. Kitayama, "Simultaneous modulation and fiber-optic transmission of $10 \mathrm{~Gb} / \mathrm{s}$ baseband and $60 \mathrm{GHz}$ band radio signals on a single wavelength," IEEE Trans. Microw. Theory Tech., vol. 49, no. 10, pp. 2013-2017, Oct. 2001.

[2] K. Ikeda, T. Kuri, and K. Kitayama, "Simultaneous three band modulation and fiber-optic transmission of $2.5 \mathrm{~Gb} / \mathrm{s}$ baseband, microwave-, and $60 \mathrm{GHz}$ band signals on a single wavelength," IEEE J. Lightw. Technol., vol. 21, no. 12, pp. 3194-3202, Dec. 2003. 
[3] A. Martinez, V. Polo, and J. Marti, "Simultaneous baseband and RF optical modulation scheme for feeding wireless and wireline heterogeneous access network," IEEE Trans. Microw. Theory Tech., vol. 49, no. 10, pp. 2018-2024, Oct. 2001.

[4] C. Lim, M. Attygalle, A. Nirmalathas, D. Novak, and R. Waterhouse, "Analysis of optical carrier-to-sideband ratio for improving transmission performance in fiber-radio links," IEEE Trans. Microw. Theory Tech., vol. 54, no. 5, pp. 2181-2187, May 2006.

[5] J. Yu, Z. Jia, L. Yi, G. K. Chang, and T. Wang, "Optical millimeter-wave generation or up-conversion using external modulator," IEEE Photon. Technol. Lett., vol. 18, no. 1, pp. 265-267, Jan. 2006.

[6] J. J. O'Reilly, P. M. Lane, R. Heidemann, and R. Hofstetter, "Optical generation of very narrow linewidth millimeter wave signals," Electron. Lett., vol. 28, no. 25, pp. 2309-2311, Dec. 3, 1992.

[7] M. Shin and P. Kumar, "Optical microwave frequency up-conversion via a frequency-doubling optoelectronic oscillator," IEEE Photon. Technol. Lett., vol. 19, no. 21, pp. 1726-1728, Nov.1 2007.

[8] W. H. Chen and W. I. Way, "Multichannel single-sideband SCM/DWDM transmission system," J. Lightw. Technol., vol. 22, no. 7, pp. 1697-1693, Jul. 2004.

[9] C. Wu and X. Zhang, "Impact of nonlinear distortion in radio over fiber systems with single-sideband and tandem single-sideband subcarrier modulations," J. Lightw. Technol., vol. 24, no. 5, pp. 2076-2090, May 2006.

[10] L. Cheng, S. Aditya, Z. Li, and A. Nirmalathas, "Generalized analysis of subcarrier multiplexing in dispersive fiber-optic links using Mach-Zehnder external modulator," J. Lightw. Technol., vol. 24, no. 6, pp. 2296-2304, Jun. 2006.

[11] T. E. Darcie, "Subcarrier multiplexing for lightwave networks and video distribution," IEEE J. Sel. Areas Commun., vol. 8, no. 7, pp. 1240-1248, Sep. 1990.

[12] G. Qi, J. Yao, J. Seregelyi, S. Paquet, and C. Bélisle, "Optical generation and distribution of continuously tunable millimeter-wave signals using an optical phase modulator," J. Lightw. Technol., vol. 23, no. 9, pp. 2687-2695, Sep. 2006.

[13] G. Qi, J. Yao, J. Seregelyi, S. Paquet, and C. Bélisle, "Generation and distribution of a wide-band continuously tunable millimeter-wave signal with an optical external modulation technique," IEEE Trans. Microw. Theory Tech., vol. 53, no. 10, pp. 3090-3097, Oct. 2006.

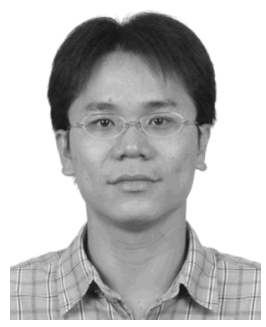

Chun-Ting Lin received the B.S. and M.S. degrees in material science and engineering from National Tsing Huang University, Hsichu, Taiwan, R.O.C., in 1997 and 2001, respectively, and the Ph.D. degree in electro-optical engineering from National Chiao-Tung University, Hsichu, Taiwan, in 2007.

His research interests are radio-over-fiber systems, optical data formats, and optoelectronic packages.

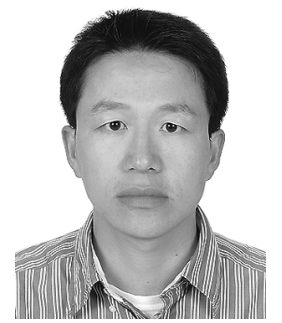

Jason (Jyehong) Chen received the B.S. and M.S degrees in electrical engineering from National Taiwan University, Taipei, Taiwan, R.O.C., in 1988 and 1990, respectively, and the Ph.D. degree in electrical engineering and computer science from the University of Maryland, Baltimore, in 1998.

$\mathrm{He}$ joined JDSU in 1998 as Senior Engineer and obtained ten U.S. patents in two years. He joined the faculty of National Chiao-Tung University, Hsinchu, Taiwan, in 2003, where he is currently an Associate Professor in the Institute of Electro-Optical Engineering and Department of Photonics.

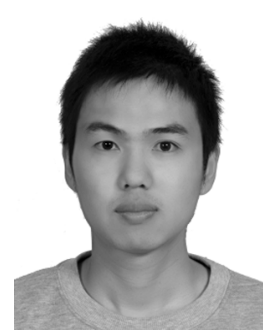

Sheng-Peng Dai received the B.S. degree in electrical engineering from National Taipei University of Technology (NTUT), Taipei, Taiwan, R.O.C., in 2006. He is currently working toward the M.S degree in electro-optical engineering at National Chiao Tung University (NCTU), Hsinchu, Taiwan.

His research interests include OFDM in radio-over-fiber and optical access networks.

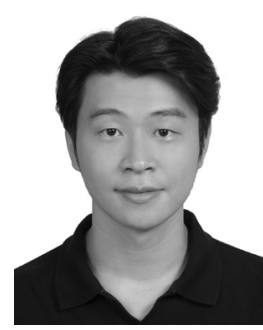

Peng-Chun Peng received the Ph.D. degree from the Institute of Electro-Optical Engineering, National Chiao Tung University, Hsinchu, Taiwan, R.O.C., in 2005.

In 2006, he joined the Department of Applied Materials and Optoelectronic Engineering, National Chi Nan University, Nantou County, Taiwan, as a Faculty Member. His research interests are optical communication devices and systems.

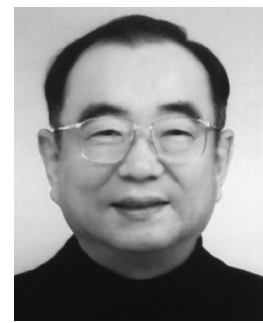

Sien Chi received the B.S.E.E. degree from the National Taiwan University, Taipei, Taiwan, R.O.C., the M.S.E.E. degree from National Chiao-Tung University, Hsinchu, Taiwan, in 1959 and 1961, respectively, and the Ph.D. degree in electro-physics from the Polytechnic Institute, Brooklyn, NY, in 1971.

From 1971 to 2004, he was a Professor at National Chiao-Tung University. From 1998 to 2001, he was the Vice President of the National Chiao-Tung University. He is currently a Chair Professor at Yuan-Ze University, Chung Li, Taiwan.

Prof. Chi is a Fellow of the Optical Society of America (OSA), His research interests are optical-fiber communications, optical solitons, optical modulation format, and optical-fiber amplifiers. 